\title{
Preschool and Kindergarten Behavior Scales - Second Edition (PKBS-2): Adaptação e Estudos Psicométricos da Versão Portuguesa
}

\author{
Preschool and Kindergarten Behavior Scales - Second Edition (PKBS-2): \\ Adaptation and Psychometric Studies of the Portuguese Version
}

\author{
Sofia Major* \& Maria João Seabra-Santos \\ Universidade de Coimbra, Coimbra, Portugal
}

\begin{abstract}
Resumo
Este artigo tem por objetivo apresentar o processo de tradução, adaptação e alguns estudos psicométricos da versão portuguesa das Preschool and Kindergarten Behavior Scales - Second Edition (PKBS-2; Merrell, 2002), uma escala de avaliação com 76 itens na sua versão original, especificamente desenvolvida para avaliar aptidões sociais e problemas de comportamento em crianças pré-escolares. A amostra normativa é composta por 1000 crianças pré-escolares avaliadas por pais e educadores/ professores. Foram efetuados estudos de tradução/adaptação e inclusão de outros itens relevantes que, após análises de itens, análise fatorial exploratória e de consistência interna, resultaram numa versão portuguesa com 80 itens. Os resultados da análise fatorial exploratória permitiram identificar três subescalas para a escala de Aptidões Sociais e duas subescalas para a de Problemas de Comportamento. As escalas apresentaram uma boa consistência interna e foram observadas intercorrelações moderadas a elevadas entre as subescalas e o resultado total. Para a estabilidade temporal, os resultados são congruentes com os da versão original, com valores moderados a elevados $(r=0,65$ a 0,90). Apesar de algumas limitações apresentadas, fica disponível uma ferramenta útil e válida na avaliação de aptidões sociais e problemas de comportamento das crianças portuguesas pré-escolares. Palavras-chave: Avaliação, pré-escolares, habilidades sociais, problemas do comportamento.
\end{abstract}

\begin{abstract}
This study aims to document the process of translation, adaptation and some psychometric studies of the Portuguese version of the Preschool and Kindergarten Behavior Scales - Second Edition (PKBS-2; Merrell, 2002), a behavior rating scale with 76 items on the original version, specifically developed to assess social skills and problem behaviors in preschool children. The normative sample comprises 1000 preschoolers rated by their parents and teachers. Studies of translation/adaptation and inclusion of other relevant items were done. After item analyses, exploratory factor analysis and internal consistency studies the Portuguese version with 80 items was developed. Exploratory factor analysis studies identified three subscales for the Social Skills Scale and two subscales for the Problem Behaviors Scale. The scales showed good internal consistency, and moderate to high correlations between subscales and the total score were observed. Results from the test-retest study are congruent with results from the original version, with moderate to high values ( $r=.65$ to .90 ). In spite of the presented limitations, it is made available a useful and valid tool for the assessment of social skills and problem behaviors of preschool Portuguese children.

Keywords: Assessment, preschoolers, social skills, behavior problems.
\end{abstract}

Durante muitos anos, as crianças pré-escolares representaram uma população negligenciada dos estudos de psicopatologia (Liu, Cheng, \& Leung, 2011), sendo a abordagem prevalecente para a identificação e intervenção de problemas de comportamento em crianças desta faixa etária "esperar para ver" (wait-and-see; Breitenstein, Hill, \& Gross, 2009). Porém, no decorrer das últimas décadas, tem-se assistido a um interesse crescente acerca do desenvolvimento/funcionamento social e emocional dos pré-escolares (Beg, Casey, \& Saunders, 2007; Carter,
* Endereço para correspondência: Faculdade de Psicologia e de Ciências da Educação, Universidade de Coimbra, Rua do Colégio Novo, 3001-802, Coimbra, Portugal. E-mail: smajor@fpce.uc.pt

O presente artigo foi elaborado a partir da tese de doutorado da primeira autora, "Avaliação de aptidões sociais e problemas de comportamento em idade pré-escolar: Retrato das crianças portuguesas", apresentada à Faculdade de Psicologia e de Ciências da Educação da Universidade de Coimbra (Portugal), com financiamento de uma bolsa de doutorado da Fundação para a Ciência e Tecnologia (SFRH/BD/29141/2006). 
Briggs-Gowan, \& Davis, 2004; Caselman \& Self, 2008; Epstein, Synhorst, Cress, \& Allen, 2009; Merrell, 1996), com a identificação e intervenção precoces nas perturbações emocionais ou comportamentais a representarem, hoje em dia, áreas em franca ascensão com forte potencial de intervenção escolar/clínica e de investigação (Bagner, Rodríguez, Black, Linares, \& Carter, 2012; Briggs-Gowan $\&$ Carter, 2008).

O aumento da atenção nesta área, por parte da psicologia do desenvolvimento e psicopatologia da criança, teve um impacto crucial na compreensão de que certos problemas de comportamento precoces predizem o funcionamento futuro (Bagner et al., 2012; Beg et al., 2007; Campbell, Shaw, \& Gilliom, 2000). Acresce que a importância de dar atenção especial aos pré-escolares com problemas de comportamento é ainda justificada atendendo ao facto de as intervenções nestas idades poderem ser mais eficazes do que na população escolar, em virtude de os comportamentos disruptivos (ou de outra natureza) estarem menos estabelecidos, sendo que é também neste período desenvolvimental que emerge o controlo comportamental (Bagner et al., 2012; Beg et al., 2007; Breitenstein et al., 2009).

No entanto, ainda que hoje em dia a identificação e atenuação de problemas socioemocionais mereça um lugar de destaque no sucesso de programas de intervenção destinados à população pré-escolar, a sua implementação tem sido travada pela falta de ferramentas de despistagem que captem os problemas mais comuns nestes contextos e nesta faixa etária mais nova (Barbarin, 2007). Em comparação com o grande número de instrumentos de avaliação desenvolvidos para utilizar com populações em idade escolar, menor atenção tem sido dada às crianças pré-escolares (Bracken, Keith, \& Walker, 1998; Carney \& Merrell, 2005; Merrell, 1995). Assim, muitos dos instrumentos de avaliação psicológica ou de despistagem utilizados com esta faixa etária apresentam sérias limitações (Denham \& Burton, 2003; Merrell, 1995) tais como: (a) uma estandardização inadequada; (b) propriedades psicométricas pobres; (c) o facto de não terem sido desenhados especificamente para trabalhar com este grupo etário mais novo; e consequentemente, (d) a circunstância de constituírem meras extensões de instrumentos desenvolvidos para crianças em idade escolar, acabando por não refletir as características únicas associadas ao desenvolvimento socioemocional desta faixa etária mais nova (Epstein et al., 2009; Merrell, 1995, 1996, 2002, 2008). No caso específico da avaliação socioemocional, apesar de alguns dos instrumentos serem adequados ao nível desenvolvimental, ainda assim pecam pelo facto de serem demasiado direcionados para perturbações específicas (e.g. psicopatologia severa) em detrimento de problemas comportamentais, sociais e emocionais com que educadores/cuidadores de crianças mais novas têm de lidar na rotina diária (Agostin \& Bain, 1997; Carney \& Merrell, 2005; Major, 2007; Merrell, 1996).

Por outro lado, embora a avaliação de défices e limitações seja fundamental para a inserção em programas de educação especial ou encaminhamento para serviços de saúde mental, tem sido salientado que o foco nesta perspetiva deficitária acaba não só por limitar a informação recolhida, mas também por enfatizar o lado negativo da criança (Epstein et al., 2009). Ou seja, a coleta de informação respeitante às potencialidades e competências da criança (e.g. habilidades sociais) revela-se um elemento chave na tarefa de avaliação psicológica com vista ao estabelecimento de protocolos interventivos, dado que o examinador obtém um retrato mais fidedigno e toma conhecimento dos recursos pessoais e ecológicos daquela, que poderão ser utilizados para alcançar as mudanças comportamentais desejadas (Achenbach \& McConaughy, 1987; Epstein et al., 2009).

Esta escassez de instrumentos destinados à avaliação de pré-escolares, vigente a nível internacional até 1980-1990, foi sendo lentamente colmatada através do aparecimento de diversas ferramentas de avaliação, tais como, questionários/escalas de avaliação, entrevistas de diagnóstico, medidas de observação estandardizadas e até baterias de avaliação neuropsicológicas especificamente destinadas para crianças pré-escolares (Angold \& Egger, 2007; Merrell, 2008). Considerando a realidade portuguesa, ao efetuar uma revisão acerca dos instrumentos disponíveis para a avaliação socioemocional da população pré-escolar (cf. Major, 2011), rapidamente se torna evidente a escassez de instrumentos nesta área. Assim, apesar da grande visibilidade e popularidade dos inventários de Achenbach enquanto instrumentos de referência na avaliação comportamental a nível internacional, em Portugal, só recentemente se encontram estudos a incidirem sobre as versões pré-escolares (informação disponível em http://www.fep. porto.ucp.pt/ASEBA/). Existem também alguns estudos realizados com crianças pré-escolares portuguesas (cf. Marzocchi et al., 2004) com o Strengths and Difficulties Questionnaire (SDQ; Goodman, 1997). Acresce ainda o desenvolvimento de raiz em português da Escala de Avaliação de Distúrbios de Comportamento da Infância (EADCI; Lopes, Machado, Pinto, Quintas, \& Vaz, 1994; Marinheiro \& Lopes, 1999).

No sentido de fazer face à carência de instrumentos de avaliação de crianças em idade pré-escolar em âmbito internacional, a disponibilização de uma ferramenta especificamente destinada à avaliação de aptidões sociais e problemas de comportamento de crianças pré-escolares, com uma aferição sólida para a população portuguesa, assume um caráter de urgência. Na revisão da literatura efetuada (Major, 2011), as Preschool and Kindergarten Behavior Scales - Second Edition (PKBS-2; Merrell, 2002) surgiram como um instrumento capaz de dar uma resposta satisfatória a esta necessidade, uma vez que constituem uma escala de avaliação do comportamento especificamente desenvolvida para ser utilizada com crianças dos 3 aos 6 anos de idade (Merrell, 2002, 2008). Assim sendo, o objetivo deste trabalho é apresentar os estudos de tradução, adaptação e validação das PKBS-2 para a população pré-escolar portuguesa. 
Major, S. \& Seabra-Santos, M. J. (2014). Preschool and Kindergarten Behavior Scales - Second Edition (PKBS-2): Adaptação e Estudos Psicométricos da Versão Portuguesa.

\section{Método}

\section{Amostra}

A amostra normativa da versão portuguesa das PKBS-2 é composta por um total de 1000 crianças, com idades entre os 3 e os 6 anos $(M=4,50$ anos, $D P=1,12)$, residentes numa das sete NUTS-II (Nomenclatura de Unidades Territoriais para Fins Estatísticos) nas quais se divide o território nacional: Norte, Centro, Lisboa, Alentejo, Algarve e Regiões Autónomas dos Açores e da Madeira; a frequentar uma instituição escolar pública $(57,7 \%)$, Instituição Particular de Solidariedade Social (IPSS) ou Obra Social $(25,7 \%)$, ou instituição particular (16,6\%). A amostra foi estratificada com um número fixo de sujeitos para as variáveis idade (250 crianças por faixa etária: 3,4 , 5 e 6 anos) e gênero ( $50 \%$ de meninos e $50 \%$ de meninas). Foi ainda estratificada atendendo às variáveis zona geográfica, meio de residência e nível de escolaridade $(50 \%$ das crianças com 6 anos frequenta o pré-escolar e 50\% a escolaridade básica (Major, 2011). O cruzamento da escolaridade e profissão dos pais das crianças (Almeida, 1988) foi utilizado como indicador do nível sócio-econômico das famílias de origem das crianças, sendo os níveis baixo $(44,6 \%)$ e médio (46,2\%) os mais representativos da amostra. Diversos critérios de inclusão foram definidos para as crianças integrarem a amostra normativa: ter o documento de consentimento de participação no estudo assinado pelos pais, ter entre 3 e 6 anos de idade, ter pais que dominem a língua portuguesa (para procederem ao preenchimento das escalas), e frequentar o jardim-de-infância/escola há pelo menos 6 semanas (recomendação do manual das PKBS-2 no sentido de os educadores/professores terem tido oportunidade de interagir com as crianças antes do preenchimento das escalas). A mãe das crianças foi o informador mais participativo/disponível para preencher as escalas em contexto familiar $(83,4 \%)$. Relativamente ao contexto escolar, um total de 131 professores participaram neste estudo ( $n=113$ em contexto de jardim-de-infância; $n$ $=18$ no primeiro ciclo do ensino básico) com uma média de oito escalas preenchidas por cada um destes informadores. Desta amostra total foi retirada uma subamostra de 80 crianças avaliadas por pais e educadores para realizar o estudo da estabilidade temporal, sendo 20 crianças por cada nível etário considerado (3-6 anos), com 50\% de meninos e $50 \%$ de meninas.

\section{Instrumento}

As PKBS-2 (Merrell, 2002) derivam de uma revisão da primeira edição (Merrell, 1994), com vista a apresentar uma versão melhorada do inventário. Compostas por 76 itens, especificamente desenvolvidos para avaliar aptidões sociais (34 itens) e problemas de comportamento (42 itens) em crianças dos 3 aos 6 anos de idade (Merrell, 2002, 2008), apesar de desenvolvidas para serem preenchidas por pais e professores, as PKBS-2 também poderão ser completadas por outros adultos com um relacionamento próximo com a criança (e.g. avós). Os itens (idênticos para o contexto familiar e escolar) são cotados em referência à observação do comportamento da criança nos últimos 3 meses, com recurso a uma escala de tipo likert de 4 pontos, de 0 "Nunca" a 3 "Muitas vezes" (Merrell, 2002). A escala de Aptidões Sociais (AS), referente a comportamentos adaptativos ou positivos, é subdividida em três subescalas: Cooperação Social (12 itens); Interação Social (11 itens); e Independência Social (11 itens). Por sua vez, a escala de Problemas de Comportamento (PC) encontra-se subdividida em duas subescalas: Problemas Exteriorizados (27 itens) e Interiorizados (15 itens), divididas ainda em subescalas suplementares de PC, derivadas de estudos de análise fatorial de segunda ordem (Merrell, 2002). O manual da versão original americana apresenta um vasto conjunto de estudos de evidência de precisão (e.g. consistência interna: $\alpha=0,90$ a 0,97 para os resultados totais; estabilidade teste-resteste: $r=0,58$ e 0,86 para três semanas e 0,69 e 0,78 para três meses, respetivamente, para os resultados totais) e validade (e.g. validade interna: estudos de análise fatorial exploratória e confirmatória; validade convergente com diversas outras medidas que avaliam construtos semelhantes, tal como o Inventário de Achenbach para professores). Várias revisões (e.g. Madle, 2005) e estudos (e.g. Herrera \& Little, 2005) comprovam as boas qualidades psicométricas e utilidade clínica destas escalas. No presente estudo utilizou-se a versão traduzida e adaptada das PKBS-2 para a população portuguesa, que sofreu várias alterações quanto ao número de itens até chegar à versão final de 80 itens.

\section{Procedimentos de Tradução e Adaptação}

Etapa 1. Após a obtenção da autorização para a tradução e adaptação das PKBS-2 para a população portuguesa junto do autor (Kenneth W. Merrell) e da editora das PKBS-2 (PRO-ED), efetuou-se um trabalho de campo com cinco educadores, um psicoterapeuta e dois professores do ensino superior especializados no trabalho com crianças pré-escolares, com o objetivo de elaborar uma listagem de aptidões sociais e problemas de comportamento relevantes nesta idade. Simultaneamente, deu-se início ao processo de tradução da versão original das PKBS-2, cuja versão portuguesa se intitulou Escalas de Comportamento para a Idade Pré-Escolar - $2^{a}$ Edição (ECIP-2).

Etapa 2. Realizou-se uma pesquisa e coleta de outros possíveis itens a acrescentar (não incluídos nas PKBS-2), a partir de outros instrumentos de avaliação de aptidões sociais e problemas de comportamento (cf. Major, 2007, 2011). Os 99 itens da versão experimental das ECIP-2 (23 itens adicionados aos 76 itens da versão original) foram analisados por profissionais especializados na área do pré-escolar, de forma a verificar a adequação da terminologia utilizada e das aptidões sociais e problemas de comportamento apresentados. Desta seleção criteriosa dos itens acabaram por ser retidos na versão experimental das ECIP-2 13 novos itens, um dos quais acrescentado à escala de $\mathrm{AS}$ e os outros 12 à escala de PC. Por conseguinte, a versão experimental das ECIP-2 era composta por um total 
de 89 itens ( 35 na escala de AS e 54 nos PC).

Etapa 3. Realizou-se um estudo-piloto com pais, educadores, amas e psicólogos, com a versão experimental das ECIP-2 (para analisar a compreensão dos itens e adequação do vocabulário). Seguiu-se o processo de retroversão, de forma a validar a tradução efetuada e a garantir a equivalência entre as duas versões, com a colaboração de uma especialista em Estudos de Tradução de Discurso Académico, que nunca teve contacto com a versão original das PKBS-2.

Etapa 4. Afinada a versão experimental das ECIP-2, procedeu-se à realização de um estudo exploratório que envolveu um total de 320 crianças dos 3 aos 6 anos, residentes na zona Centro do país, sendo cada uma avaliada pelos pais e respetivo educador/professor $(N=640 \mathrm{ECIP}-2$ recolhidas; Major, 2007; Major, Seabra-Santos, \& Merrell, 2011). Os resultados obtidos para os coeficientes de consistência interna com os métodos alfa e split-half, para os totais da escala de AS, oscilaram entre 0,85 e 0,94 , e para os PC entre 0,89 e 0,97 . Os primeiros estudos de análise fatorial exploratória realizados com os 35 itens da escala de AS e os 54 itens da escala de PC (ECIP-2 preenchidas por pais, por educadores/professores e para a totalidade de escalas recolhidas) levaram à obtenção de uma estrutura de três fatores para a escala de AS (Cooperação Social, Interação Social e Independência Social) e dois fatores para os PC (PC Exteriorizados e PC Interiorizados). Os 13 itens adicionados na versão portuguesa das PKBS-2 saturaram nos fatores esperados com base no referencial teórico (Major, 2007), daí a manutenção dos 89 itens (tradução dos 76 itens das PKBS-2 mais os 13 itens adicionados aquando dos estudos de tradução e adaptação) das ECIP-2 no estudo normativo, com a introdução de ligeiros ajustamentos em termos da formulação de seis itens com base nos resultados do estudo exploratório (Major, 2007) e nalgumas sugestões de pais e educadores/professores (cf. Major, 2011).

\section{Procedimento de Coleta dos Dados}

Num primeiro momento efetuaram-se os pedidos de autorizações necessários para a realização do estudo e coleta da amostra junto das entidades nacionais competentes: Comissão Nacional de Proteção de Dados, Direções Regionais de Educação de todo o país e Direção Geral da Inovação e Desenvolvimento Curricular. Posteriormente procedeu-se à seleção aleatória das instituições pré-escolares que iriam participar no estudo, sendo as instituições do primeiro ciclo do ensino básico selecionadas por proximidade física face às instituições pré-escolares. Os vários diretores e/ou coordenadores das instituições escolares selecionadas foram contactados por telefone, carta ou e-mail para apresentação do projeto e obtenção do seu consentimento para a participação no estudo. Uma vez definido o número de crianças por instituição e efetuada a seleção aleatória das crianças, foram entregues aos educadores/professores os exemplares das ECIP-2 a preencher, bem como um envelope para os pais de cada criança a incluir na amostra. $\mathrm{O}$ envelope entregue aos pais continha uma carta de apresentação do projeto, em que era solicitada a sua colaboração através do preenchimento das escalas (sendo explicado que o educador também iria preencher a mesma escala e pedida a sua autorização para este segundo preenchimento), uma folha de consentimento informado a ser assinada pelos pais e um exemplar das ECIP-2. Os pais devolviam as ECIP-2 e a folha de consentimento ao educador dentro do mesmo envelope, mas agora fechado (para garantir a confidencialidade das respostas dos pais). O tempo médio de preenchimento foi de cerca de 13 minutos em contexto familiar e 10 minutos em contexto escolar $(M=12,76$, $D P=5,92$ e $M=10,25, D P=4,42$, respetivamente). A taxa de retorno das escalas entregues para preenchimento foi de $87,8 \%$ e de $100 \%$, respetivamente pelos pais e em contexto escolar (uma vez que obtido o consentimento dos pais para a participação no estudo, nenhum dos educadores/ professores se opôs ao preenchimento das ECIP-2). Para o estudo da estabilidade temporal solicitou-se a 80 dos pais e educadores que compõem a amostra normativa que, após terem preenchido as ECIP-2 (linha de base), procedessem ao seu preenchimento noutros dois momentos: 3 semanas (reteste 1) e 3 meses mais tarde (reteste 2).

\section{Resultados}

Validade Interna - Análise Fatorial Exploratória 89 itens

Procedeu-se à averiguação do cumprimento de diversos pressupostos para a realização dos estudos de análise fatorial exploratória (AFE) com a versão experimental das ECIP-2. No que diz respeito à dimensão da amostra, o facto de 1000 sujeitos terem sido avaliados para os 89 itens da versão experimental das ECIP-2 responde até às abordagens variável-sujeito mais conservadoras de 10 sujeitos por cada item (Nunnally, 1978). Seguindo a opção do autor das PKBS-2 (Merrell, 2002) e de acordo com as análises apresentadas no manual original, os estudos de AFE apresentados focam-se na totalidade de inventários recolhidos $(N=2000)$. Porém, foram igualmente efetuados estudos para os protocolos recolhidos em contexto familiar e escolar de forma independente, sendo os respetivos resultados congruentes com os realizados para a totalidade de protocolos, o que justifica a utilização de uma versão única das ECIP-2 para ser preenchida em ambos os contextos. Quanto à fatoriabilidade dos dados, os valores do Teste de Adequação da Amostra de Kaiser-Meyer-Olkin (KMO; 0,95 e 0,98, para as AS e PC, respetivamente) ultrapassam largamente o limite mínimo recomendado de 0,60 (Tabachnick \& Fidell, 2001) e o Teste de Esfericidade de Bartlett atinge a significância estatística $(p<$ $0,001)$. Para os 35 itens das AS, com recurso ao critério de Kaiser, seriam retidos seis componentes com Eigenvalues superiores ou iguais a 1 , que explicam $54,11 \%$ da variância. No entanto, a análise do scree test de Cattell (Tabachnick \& Fidell, 2001), tornou mais clara a retenção de três componentes (solução que corresponde à estrutura das PKBS-2). Já para os 54 itens dos PC, segundo o critério de Kaiser seriam retidos sete componentes que explicavam 
$58,73 \%$ da variância, com o scree test a apontar para um número de componentes a reter não superior a três (cf. Major, 2011). O cruzamento dos estudos iniciais de AFE com retenção dos itens no respetivo fator cujas cargas fatoriais apresentavam um valor mínimo de 0,30 , com os estudos de correlações entre itens e os primeiros estudos de consistência interna apontaram para a necessidade de remover alguns itens. Assim, à semelhança do que é feito noutros estudos (e.g. Epstein et al., 2009) foi estabelecido um conjunto de critérios para se proceder à eliminação de itens (e.g. itens com sobreposição de conteúdo com itens já incluídos nas PKBS-2, itens com correlações todas inferiores a 0,30 com os restantes itens, itens com conteúdo que possa levar a interpretações distintas em contexto familiar e escolar; cf. Major, 2011). Esta seleção levou à eliminação de nove itens da versão experimental das ECIP-2 (item 24 , original da escala de AS e 33 , original da escala de PC, e itens 43, 44, 45, 48, 49, 53 e 54, todos adicionados à versão experimental), ficando a versão final das ECIP-2 composta por 80 itens (34 na escala de AS e 46 na escala de PC). Dos 34 itens de AS das PKBS-2, o item 24 ("Procura consolo no adulto quando se magoa") foi eliminado por ser o único item desta escala com correlações negativas e/ou não significativas com outros itens da mesma escala e por apresentar uma correlação item-total corrigido inferior a 0,30, nos estudos de consistência interna. Acresce que este item surge nas PKBS-2 na subescala de Interação Social, sendo que o item 35, adicionado à versão portuguesa, saturou nessa mesma escala (compensando, em termos numéricos, a eliminação do item 24). Quanto aos 42 itens de PC das PKBS-2, o item 33 ("Comporta-se como uma criança mais nova”) foi removido pelo facto de o seu conteúdo se pautar por alguma falta de especificidade, ao representar uma caracterização global do comportamento da criança, mais do que um comportamento específico. Por outro lado, foi o único item das PKBS-2 que, nos estudos iniciais de AFE apresentou uma saturação muito próxima em ambas as subescalas de PC $(0,37$ e 0,34$)$.

Por limitações de espaço, os estudos relativos às subescalas suplementares de PC serão apresentados num trabalho posterior.
Validade Interna - Análise Fatorial Exploratória 80 itens

Procedeu-se à análise de componentes principais aos 34 itens da escala de AS. O resultado do Teste de Esfericidade de Bartlett atinge a significância estatística, $\chi^{2}(561)$ $=26749,51, p<0,001$, e o KMO obtém o valor de 0,95. A rotação com solução oblíqua pelo método Direct Oblimin convergiu em 10 iterações, para uma estrutura de três componentes. A análise do conteúdo dos itens marcadores levou a ligeiros ajustamentos na denominação dos componentes considerados, para uma classificação mais clara dos itens aí abrangidos, procurando manter sempre que possível a denominação original. A Tabela 1 indica que o primeiro componente abrange um total de 13 itens (cargas fatoriais entre 0,38-0,73) referentes a comportamentos de Independência Social/Assertividade, assim designado por integrar comportamentos de iniciativa social das crianças, mas também de alguma assertividade nas suas relações sociais (10 itens são comuns com as PKBS-2). O segundo componente, Cooperação/Ajustamento Social, abarca 11 itens (cargas fatoriais entre 0,31-0,83) relacionados com comportamentos de cooperação valorizados na relação com adultos (11 itens são comuns com as PKBS-2). Para a versão portuguesa foi adicionado o termo "ajustamento", uma vez que, para além de comportamentos de cooperação, também estão representados comportamentos ajustados às normas sociais. Finalmente, o terceiro componente denominado de Interação Social/Empatia, mais direcionado para as relações com os pares no que diz respeito ao desenvolvimento e manutenção de amizades e participação social positiva (daí a adição do termo "empatia”), é composto por 10 itens (cargas fatoriais entre 0,33-0,83) na versão portuguesa (11 itens na versão americana). No caso do item 2, apesar deste apresentar uma saturação ligeiramente mais elevada no componente de Independência Social/Assertividade do que na Cooperação/Ajustamento Social (0,34 vs. 0,31, respetivamente), o facto de também estar incluído neste componente nas PKBS-2, a reduzida discrepância entre as duas saturações e o conteúdo do item levaram à sua manutenção no segundo componente. Os mesmos critérios serviram de base à fixação do item 14 no componente de Interação Social/Empatia.

Tabela 1

Matriz de Componentes Rodada, Comunalidades, Variância Explicada e Alfa de Cronbach: Aptidões Sociais - 34 Itens

\begin{tabular}{|c|c|c|c|c|c|c|c|}
\hline & \multirow{2}{*}{ Item } & \multicolumn{3}{|c|}{ Componente } & \multirow{2}{*}{$h^{2}$} & \multirow{2}{*}{$\begin{array}{c}\text { Correlação } \\
\text { item-total }\end{array}$} & \multirow{2}{*}{$\begin{array}{c}\alpha \text { item } \\
\text { eliminado }\end{array}$} \\
\hline & & 1 & 2 & 3 & & & \\
\hline 9. & Faz amigos... & 0,73 & & & 0,53 & 0,53 & 0,92 \\
\hline & Brinca... & 0,70 & & & 0,49 & 0,44 & 0,93 \\
\hline & Adapta-se bem... & 0,66 & & & 0,44 & 0,47 & 0,92 \\
\hline 11. & É convidado(a)... & 0,64 & & & 0,41 & 0,53 & 0,92 \\
\hline & Mostra-se confiante... & 0,62 & & & 0,38 & 0,46 & 0,92 \\
\hline 3. & Sorri... & 0,58 & & & 0,34 & 0,44 & 0,93 \\
\hline 6. & É aceite... & 0,58 & & & 0,34 & 0,49 & 0,92 \\
\hline
\end{tabular}




\begin{tabular}{|c|c|c|c|c|c|c|c|}
\hline 21. & Convida outras crianças... & 0,55 & & & 0,30 & 0,58 & 0,92 \\
\hline 13. & Na rotina diária consegue separar-se... & 0,45 & & & 0,20 & 0,34 & 0,93 \\
\hline 19. & Tem competências... & 0,44 & & & 0,19 & 0,55 & 0,92 \\
\hline 8. & Tenta resolver... & 0,41 & & & 0,17 & 0,48 & 0,92 \\
\hline 14. & Participa nas conversas... & 0,40 & & $-0,33$ & 0,27 & 0,54 & 0,92 \\
\hline 12. & Usa o tempo livre... & 0,38 & & & 0,14 & 0,52 & 0,92 \\
\hline 1. & Trabalha ou brinca... & 0,38 & & & 0,14 & 0,32 & 0,93 \\
\hline 2. & É cooperante... & 0,34 & 0,31 & & 0,22 & 0,55 & 0,92 \\
\hline 23. & Cumpre... & & 0,83 & & 0,69 & 0,49 & 0,92 \\
\hline 7. & Segue instruções... & & 0,76 & & 0,58 & 0,48 & 0,92 \\
\hline 29. & Aceita decisões... & & 0,76 & & 0,58 & 0,46 & 0,92 \\
\hline 30. & Espera pela sua vez... & & 0,72 & & 0,52 & 0,47 & 0,92 \\
\hline 22. & Arruma... & & 0,72 & & 0,52 & 0,39 & 0,93 \\
\hline 10. & Demonstra auto-controlo... & & 0,63 & & 0,40 & 0,54 & 0,92 \\
\hline 28. & Quando necessário cede... & & 0,48 & $-0,39$ & 0,38 & 0,61 & 0,92 \\
\hline 16. & Senta-se e ouve... & & 0,46 & & 0,21 & 0,43 & 0,93 \\
\hline 25. & Partilha... & & 0,40 & & 0,16 & 0,48 & 0,92 \\
\hline 32. & Responde apropriadamente... & & 0,38 & & 0,14 & 0,55 & 0,92 \\
\hline 33. & É sensível... & & & $-0,83$ & 0,69 & 0,52 & 0,92 \\
\hline 20. & Consola... & & & $-0,78$ & 0,61 & 0,61 & 0,92 \\
\hline 5. & Tenta compreender... & & & $-0,69$ & 0,48 & 0,60 & 0,92 \\
\hline 34. & Demonstra afeto... & & & $-0,67$ & 0,45 & 0,56 & 0,92 \\
\hline 35. & Oferece ajuda... & & & $-0,66$ & 0,44 & 0,65 & 0,92 \\
\hline 17. & Defende os direitos... & & & $-0,57$ & 0,32 & 0,58 & 0,92 \\
\hline 27. & Pede desculpa... & & 0,37 & $-0,55$ & 0,44 & 0,56 & 0,92 \\
\hline 26. & Defende... & & & $-0,48$ & 0,23 & 0,44 & 0,93 \\
\hline 15. & Pede ajuda... & & & $-0,45$ & 0,20 & 0,38 & 0,93 \\
\hline & \% Variância Total & 7,37 & 6,26 & 7,31 & & & \\
\hline & $\alpha$ Amostra Total & 0,85 & 0,87 & 0,87 & & & \\
\hline & $\alpha$ Contexto Familiar & 0,76 & 0,81 & 0,77 & & & \\
\hline & $\alpha$ Contexto Escolar & 0,89 & 0,90 & 0,90 & & & \\
\hline
\end{tabular}

Nota. Para os itens com dupla carga fatorial, encontra-se a itálico aquela que traduz a opção feita quanto à inclusão num dos componentes.

No que diz respeito aos 46 itens da escala de PC, para a fatoriabilidade dos dados destaca-se o valor do KMO $(0,97)$ e Teste de Esfericidade de Bartlett a atingir a significância estatística, $\chi^{2}(1035)=53578,72, p<0,001$. A análise dos componentes principais, com solução oblíqua pelo método de rotação Direct Oblimin convergiu em quatro iterações, para uma estrutura de dois componentes. A análise da Tabela 2 indica que o primeiro componente, PC Exteriorizados, contém 29 itens muito direcionados para comportamentos disruptivos, agressivos e de atividade excessiva (cargas fatoriais entre 0,45 e 0,81 ). Ao comparar os itens incluídos na Tabela 2 com os das PKBS-2, excetuando os itens 46, 50 e 52 (adicionados às ECIP-2), existe uma notável sobreposição entre as duas versões, com uma única modificação: o item 37 que deixou de integrar os PC Exteriorizados para, na versão portuguesa, integrar os PC Interiorizados. O segundo componente refere-se a PC Interiorizados, com 17 itens muito associados a características comportamentais de isolamento e ansiedade (cargas de 0,37-0,73). Existe novamente uma elevada sobreposição com os itens incluídos nas PKBS-2 dado que, para além dos itens adicionados para as ECIP-2 (itens 47 e 51), apenas se encontra a alteração anteriormente referida do item 37. 
Major, S. \& Seabra-Santos, M. J. (2014). Preschool and Kindergarten Behavior Scales - Second Edition (PKBS-2): Adaptação e Estudos Psicométricos da Versão Portuguesa.

Tabela 2

Matriz de Componentes Rodada, Comunalidades e Variância Explicada e Alfa de Cronbach: Problemas de Comportamento - 46 Itens

\begin{tabular}{|c|c|c|c|c|c|c|}
\hline & \multirow{2}{*}{ Item } & \multicolumn{2}{|c|}{ Componente } & \multirow{2}{*}{$h^{2}$} & \multirow{2}{*}{$\begin{array}{c}\text { Correlação } \\
\text { item-total }\end{array}$} & \multirow{2}{*}{$\begin{array}{c}\alpha \text { item } \\
\text { eliminado }\end{array}$} \\
\hline & & 1 & 2 & & & \\
\hline 16. & Desobedece... & 0,81 & & 0,66 & 0,67 & 0,96 \\
\hline 25. & É irrequieto(a)... & 0,81 & & 0,66 & 0,66 & 0,96 \\
\hline 20. & É extremamente activo(a)... & 0,78 & & 0,61 & 0,63 & 0,96 \\
\hline 22. & Desafia... & 0,78 & & 0,61 & 0,67 & 0,96 \\
\hline 39. & Perturba... & 0,78 & & 0,61 & 0,67 & 0,96 \\
\hline 42. & Aborrece... & 0,77 & & 0,59 & 0,72 & 0,96 \\
\hline 14. & Tira coisas... & 0,77 & & 0,59 & 0,65 & 0,96 \\
\hline 13. & Grita... & 0,76 & & 0,58 & 0,69 & 0,96 \\
\hline 11. & É fisicamente agressivo(a)... & 0,75 & & 0,56 & 0,66 & 0,96 \\
\hline 6. & Faz barulhos... & 0,74 & & 0,55 & 0,64 & 0,96 \\
\hline 46. & Não sabe esperar... & 0,74 & & 0,55 & 0,68 & 0,96 \\
\hline 1. & Age impulsivamente... & 0,74 & & 0,55 & 0,59 & 0,96 \\
\hline 50. & Envolve-se... & 0,72 & & 0,52 & 0,59 & 0,96 \\
\hline 41. & É facilmente provocado(a)... & 0,72 & & 0,52 & 0,70 & 0,96 \\
\hline 19. & Tudo tem de ser... & 0,71 & & 0,50 & 0,69 & 0,96 \\
\hline 7. & Enerva-se facilmente... & 0,71 & & 0,50 & 0,68 & 0,96 \\
\hline 3. & Faz troça... & 0,67 & & 0,45 & 0,59 & 0,96 \\
\hline 52. & Não muda... & 0,65 & & 0,42 & 0,65 & 0,96 \\
\hline 21. & Procura vingar-se... & 0,65 & & 0,42 & 0,65 & 0,96 \\
\hline 31. & Tem um comportamento... & 0,65 & & 0,42 & 0,69 & 0,96 \\
\hline 34. & Destrói objectos... & 0,64 & & 0,41 & 0,62 & 0,96 \\
\hline 29. & Ameaça... & 0,61 & & 0,37 & 0,61 & 0,96 \\
\hline 26. & Chama nomes... & 0,58 & & 0,34 & 0,56 & 0,96 \\
\hline 40. & Diz... & 0,56 & & 0,31 & 0,57 & 0,96 \\
\hline 8. & Requer toda a atenção... & 0,54 & & 0,29 & 0,63 & 0,96 \\
\hline 15. & Tem dificuldades... & 0,53 & & 0,28 & 0,58 & 0,96 \\
\hline 35. & É mal-humorado(a)... & 0,53 & & 0,28 & 0,66 & 0,96 \\
\hline 32. & Tem ciúmes... & 0,46 & & 0,22 & 0,58 & 0,96 \\
\hline 10. & Não partilha... & 0,45 & & 0,20 & 0,57 & 0,96 \\
\hline 30. & Aparenta estar infeliz... & & 0,73 & 0,53 & 0,46 & 0,96 \\
\hline 18. & É apreensivo(a)... & & 0,72 & 0,52 & 0,40 & 0,96 \\
\hline 28. & Afasta-se... & & 0,71 & 0,50 & 0,48 & 0,96 \\
\hline 47. & Parece apático(a)... & & 0,70 & 0,49 & 0,45 & 0,96 \\
\hline 17. & Tem dificuldade... & & 0,69 & 0,48 & 0,42 & 0,96 \\
\hline 12. & Evita brincar... & & 0,65 & 0,42 & 0,48 & 0,96 \\
\hline 2. & Adoece... & & 0,63 & 0,40 & 0,31 & 0,96 \\
\hline 51. & Não sabe divertir-se... & & 0,59 & 0,35 & 0,41 & 0,96 \\
\hline 27. & É difícil de consolar... & & 0,59 & 0,35 & 0,53 & 0,96 \\
\hline
\end{tabular}




\begin{tabular}{|c|c|c|}
\hline 24. & Resiste... & \\
\hline 9. & É ansioso(a)... & \\
\hline 23. & Queixa-se... & \\
\hline 5. & Agarra-se... & \\
\hline 38. & As outras crianças aproveitam-se... & \\
\hline 37. & Choraminga... & \\
\hline 36. & É extremamente sensível... & \\
\hline 4 & Não responde... & \\
\hline & \% Variância Total & 15,92 \\
\hline & $\alpha$ Amostra Total & 0,96 \\
\hline & $\alpha$ Contexto Familiar & 0,94 \\
\hline & $\alpha$ Contexto Escolar & 0,97 \\
\hline
\end{tabular}

Validade Interna - Intercorrelações entre Escalas e Resultado Total

A análise dos resultados apresentados na Tabela 3, referentes às relações entre subescalas e respetivos resultados totais das ECIP-2 $(p<0,01)$ calculadas através do coeficiente de correlação de Pearson, permite-nos concluir que, para a escala de AS, as intercorrelações entre escalas oscilam entre 0,51 e 0,66 e entre $0,81-0,86$ com o resul- tado total. Para a escala de PC, as intercorrelações com o resultado total $(r=0,79-0,96)$ apresentam uma maior amplitude face às AS, com uma correlação de 0,57 entre as duas subescalas. Quanto às correlações entre os resultados das duas escalas, estas são todas negativas e com uma intensidade fraca a moderada. A correlação entre o total da escala de AS e de PC é de $-0,49$, muito próxima do valor obtido para as PKBS-2 $(r=-0,54)$ e indica que os dois resultados partilham $24 \%$ da sua variância.

Tabela 3

Intercorrelações entre Subescalas e Resultados Totais ECIP-2 $(N=2000)$

\begin{tabular}{llllllll}
\hline & \multicolumn{3}{c}{ Escala de AS } & \multicolumn{3}{c}{ Escala de PC } \\
\cline { 2 - 8 } Resultados ECIP-2 & CAS & ISE & ISA & TAS & PE & PI & TPC \\
\hline Escala de AS & & & & & & & \\
Cooperação/Ajustamento Social & & & & & & & \\
Interação Social/Empatia & 0,54 & & & & & & \\
Independência Social/Assertividade & 0,51 & 0,66 & & & & & \\
Total Aptidões Sociais & 0,81 & 0,86 & 0,86 & & & & \\
Escala de PC & & & & & & & \\
Problemas Exteriorizados & $-0,67$ & $-0,20$ & $-0,21$ & $-0,43$ & & \\
Problemas Interiorizados & $-0,39$ & $-0,32$ & $-0,52$ & $-0,48$ & 0,57 & \\
Total Problemas Comportamento & $-0,64$ & $-0,27$ & $-0,34$ & $-0,49$ & 0,96 & 0,79
\end{tabular}

Nota . CAS=Cooperação/Ajustamento Social; ISE=Interação Social/Empatia; ISA=Independência Social/Assertividade; TAS=Total Aptidões Sociais; PE=Problemas Exteriorizados; PI=Problemas Interiorizados; TPC=Total Problemas Comportamento. Todos os coeficientes são estatisticamente significativos a $p<0,01$ (bicaudal).

\section{Fiabilidade - Consistência Interna}

No sentido de avaliar a homogeneidade dos itens das ECIP-2, foram calculados os índices de consistência in- terna (alfa de Cronbach) para as três subescalas de AS e duas subescalas de PC, bem como, para os dois resultados totais das escalas, considerando a totalidade de protocolos 
das ECIP-2 recolhidos $(N=2000)$ e separadamente para os preenchimentos em contexto familiar ou escolar $(N=1000$, por contexto; cf. Tabelas 1 e 2). Obtiveram-se níveis de precisão para os totais das escalas num valor considerado excelente (R. B. Kline, 1998) de 0,90 ou superior $(\alpha=$ $0,93,0,88$ e 0,95 para as AS e $\alpha=0,96,0,95$ e 0,97 para os PC; considerando a totalidade de protocolos preenchidos, contexto familiar e contexto escolar, respetivamente), sendo que para as várias subescalas são ultrapassados os valores mínimos considerados aceitáveis (P. Kline, 2000; Nunnally, 1978), situando-se todos acima de 0,76. Considerando a totalidade dos protocolos, todos os valores de consistência interna para as subescalas de AS e PC, se encontram acima de 0,85 , não existindo qualquer item cuja eliminação levasse a um aumento do valor do coeficiente de consistência interna, para os totais das escalas de AS (correlações item-total corrigidas entre 0,32 e 0,65) e PC (correlações item-total corrigidas entre 0,31 e 0,72; cf. Tabelas 1 e 2). Para os protocolos respondidos em contexto familiar, os resultados são inferiores aos alcançados considerando a totalidade da amostra (tal como nas PKBS-2), com valores para as subescalas a oscilarem entre $0,76 \mathrm{e}$ 0,94 . Para o contexto escolar, as subescalas apresentam valores de consistência interna muito satisfatórios, entre 0,89 e 0,97 , e superiores, ainda, aos obtidos para as ECIP2 preenchidas pelos pais e considerando a totalidade dos protocolos (cf. Tabelas 1 e 2).

\section{Fiabilidade - Estabilidade Temporal}

$\mathrm{Na}$ Tabela 4 são apresentados os resultados do estudo de fiabilidade teste-reteste (ou estabilidade temporal), operacionalizados através do cálculo dos coeficientes de correlação de Pearson entre a avaliação da linha de base e os dois momentos seguintes ( 3 semanas e 3 meses), para os contextos familiar e escolar. Todas as correlações obtidas são estatisticamente significativas $(p<0,01)$, num nível moderado a elevado. Os resultados das ECIP-2, quer sejam preenchidas em contexto familiar ou escolar, tendem a apresentar uma estabilidade mais elevada para os PC em comparação com as AS. Relativamente aos resultados totais das escalas, com exceção da escala de AS quando preenchida pelos pais $(r=0,72$ para 0,75$)$, assiste-se a um ligeiro decréscimo do valor do coeficiente de correlação quando se passa de 3 semanas para 3 meses de intervalo de tempo decorrido desde a avaliação da linha de base. No que diz respeito às subescalas, ainda que alguns coeficientes de correlação apresentem uma redução das 3 semanas para os 3 meses (PC Interiorizados preenchimento em contexto escolar), outros apresentam um valor estável nos dois momentos (PC Exteriorizados preenchimento em contexto escolar), e outros ainda, chegam a apresentar ligeiros aumentos (Interação Social preenchimento em contexto familiar).

Tabela 4

Coeficientes de Estabilidade Temporal Contextos Familiar e Escolar (3 Semanas e 3 Meses)

\begin{tabular}{lcccc}
\hline & \multicolumn{2}{c}{ Contexto familiar } & \multicolumn{2}{c}{ Contexto escolar } \\
\cline { 2 - 5 } Resultados ECIP-2 & $\begin{array}{c}\text { Reteste } \\
\text { 3 semanas }\end{array}$ & $\begin{array}{c}\text { Reteste } \\
3 \text { meses }\end{array}$ & $\begin{array}{c}\text { Reteste } \\
\text { 3 semanas }\end{array}$ & $\begin{array}{c}\text { Reteste } \\
3 \text { meses }\end{array}$ \\
\hline Escala de AS & & & & \\
Cooperação/Ajustamento Social & 0,74 & 0,69 & 0,81 & 0,75 \\
Interação Social/Empatia & 0,70 & 0,74 & 0,84 & 0,77 \\
Independência Social/Assertividade & 0,67 & 0,68 & 0,82 & 0,82 \\
Total Aptidões Sociais & 0,72 & 0,75 & 0,87 & 0,81 \\
Escala de PC & & & & \\
Problemas Exteriorizados & 0,88 & 0,87 & 0,89 & 0,89 \\
Problemas Interiorizados & 0,87 & 0,82 & 0,77 & 0,65 \\
Total Problemas Comportamento & 0,90 & 0,87 & 0,87 & 0,84
\end{tabular}

Nota. Todos os coeficientes são estatisticamente significativos a $p<0,01$ (bicaudal).

\section{Discussão e Conclusões}

O presente estudo teve por objetivo apresentar os estudos de tradução, adaptação e validação das PKBS-2 para a população portuguesa. A opção pelas PKBS-2 (Merrell, 2002) em detrimento de outros inventários comportamentais justifica-se por: não se focarem apenas nas fragilidades da criança (problemas de comportamento); recorrerem ao mesmo conjunto de itens para as avaliações efetuadas em contexto familiar e escolar; terem sido desenvolvidas especificamente para a idade pré-escolar; e apresentarem boas propriedades psicométricas, evidenciadas nos estudos apresentados no manual da versão original (Merrell, 2002), e em diversas revisões e estudos empíricos realizados des- 
de a sua publicação (e.g. Herrera \& Little, 2005; Madle, 2005). O processo de tradução obedeceu a diversas fases, de forma a garantir a manutenção do sentido/conteúdo dos itens originais e a sua clara compreensão por parte dos respondentes portugueses. Foram tomadas as devidas precauções e seguidas as recomendações aquando do desenvolvimento e tradução de um instrumento de avaliação (e.g. tradução e retroversão) até se alcançar a versão das ECIP-2 para a coleta da amostra normativa.

Ao nível dos estudos psicométricos, no que diz respeito à evidência de validade com base na estrutura interna das ECIP-2, podemos afirmar que, apesar de afastamentos pontuais em relação à composição fatorial das PKBS-2, encontra-se uma elevada sobreposição entre as duas versões. Por conseguinte, apesar da eliminação de dois itens da versão original e de seis itens terem sido adicionados à versão portuguesa, podemos afirmar que os resultados obtidos são consistentes com os do estudo exploratório anteriormente realizado (Major, 2007) e com os que constam do manual das PKBS-2, confirmando a divisão das ECIP-2 em duas escalas: Aptidões Sociais (com três subescalas) e Problemas de Comportamento (com uma estrutura bifatorial). $\mathrm{O}$ facto de se terem sido realizados estudos não só com a totalidade dos questionários recolhidos $(N=2000)$, mas também com os questionários preenchidos em contextos diferentes (casa e escola) reforça a replicabilidade e validade da estrutura fatorial obtida. Ainda no âmbito dos estudos relacionados com a evidência de validade interna, os resultados obtidos para o estudo da relação entre subescalas e resultados totais das ECIP-2 levam-nos a concluir que, tal como nas PKBS-2, as correlações entre os resultados das escalas de AS e de PC são baixas a moderadas e numa direção negativa, o que é justificável atendendo ao facto de a presença de problemas de comportamento interferir com o desenvolvimento adequado de aptidões sociais (Merell \& Gimpel, 1998). Por outro lado, e como expectável, as correlações são superiores entre as subescalas e a respetiva escala em que estão incluídas (AS ou PC) do que com qualquer um dos resultados da outra escala. Este conjunto de resultados é favorável à solidez/ adequação da relação entre as várias escalas e subescalas obtidas nos estudos de AFE e congruentes com os valores apontados no manual das PKBS-2 (Merrell, 2002).

No que diz respeito à análise da fiabilidade, os resultados obtidos para os estudos de consistência interna e considerando quer cada um dos contextos estudados, quer o total de ECIP-2 preenchidas, apontam para uma boa homogeneidade da versão portuguesa, com os totais das escalas de AS e PC classificáveis no patamar de excelência (segundo a classificação proposta por R. B. Kline, 1998), mas também resultados muito ajustados para as várias subescalas. Numa análise mais pormenorizada, o estudo das correlações item-total corrigidas e dos valores dos coeficientes alfa de Cronbach, caso algum dos itens fosse removido, reforçam os resultados anteriores. Quanto aos estudos de estabilidade temporal, infere-se que os resultados das ECIP-2, quer sejam preenchidas em contexto familiar ou escolar, tendem a apresentar uma estabilidade mais elevada para os PC do que para as AS. Esta situação também se verificou nas PKBS-2 e o autor aponta a menor estabilidade dos comportamentos sociais das crianças pré-escolares como uma possível justificação para os resultados encontrados (Merrell, 2002). Neste contexto, Merrell (2008) salienta que os coeficientes de estabilidade temporal com valores moderados são a regra, mais do que a exceção, nas escalas destinadas a avaliar comportamentos em idades precoces, uma vez que existe uma maior variabilidade do construto a avaliar ao longo do tempo, devido a alterações reais dos comportamentos ou a fatores desenvolvimentais (P. Kline, 2000). Esta característica é inerente à avaliação de pré-escolares, mais do que indicadora de problemas com o instrumento em questão (Carney \& Merrell, 2005). Ainda assim, os resultados relativamente estáveis constituem mais um ponto a favor da evidência de precisão dos resultados obtidos e da estabilidade do construto avaliado pelas ECIP-2.

Em termos práticos, encontramos implicações significativas resultantes deste trabalho. Este estudo veio trazer um contributo positivo à avaliação socioemocional de crianças pré-escolares em Portugal, ficando disponível um instrumento de avaliação socioemocional, criado de raiz para esta faixa etária. A possibilidade de as ECIP-2 poderem ser utilizadas nos dois contextos com mais impacto na vida da criança (familiar e escolar), associadas à robustez psicométrica deste instrumento, fazem das ECIP-2 uma ferramenta capaz de avaliar importantes construtos relativos ao funcionamento socioemocional da criança e, através de uma medida única, fornecer informação acerca das potencialidades (aptidões sociais) e limitações (problemas de comportamento) da criança. Ao disponibilizar um instrumento desta natureza, devidamente adaptado para a população portuguesa, este estudo representa, igualmente, um pequeno contributo metodológico ao nível da investigação futura a desenvolver no país com crianças pré-escolares. Este trabalho contém algumas limitações que merecem ser examinadas, nomeadamente, os resultados dos estudos de AFE, que não são tão positivos quão desejável para os itens da escala de AS. Neste sentido, apontamos diversas sugestões para estudos futuros tais como, a realização de estudos de análise fatorial confirmatória agrupando os itens em item testlets, de forma a reduzir o seu número e a criar um construto mais consistente; a replicação destes estudos com pais e educadores; a adaptação das ECIP-2 para outros países de língua portuguesa; e a publicação de um manual das ECIP-2.

\section{Referências}

Achenbach, T. M., \& McConaughy, S. H. (1987). Empirically based assessment of child and adolescent psychopathology: Practical applications. Developmental Clinical Psychology and Psychiatry (Vol. 13). Newbury Park, CA: Sage.

Agostin, T. M., \& Bain, S. K. (1997). Predicting early school success with developmental and social skills screeners. Psychology in the Schools, 34(3), 219-228. 
Almeida, L. S. (1988). O raciocínio diferencial dos jovens portugueses: Avaliação, desenvolvimento e diferenciação. Porto, Portugal: Instituto Nacional de Investigação Científica.

Angold, A., \& Egger, H. L. (2007). Preschool psychopathology: Lessons for the lifespan. Journal of Child Psychology and Psychiatry, 48(10), 961-966. doi:10.1111/j.14697610.2007.01832.x

Bagner, D. M., Rodríguez, G. M., Blake, C. A., Linares, D., \& Carter, A. S. (2012). Assessment of behavioral and emotional problems in infancy: A systematic review. Clinical Child and Family Psychology Review, 15, 113-128. doi:10.1007/ s10567-012-0110-2

Barbarin, O. A. (2007). Mental health screening of preschool children: Validity and reliability of ABLE. American Journal of Orthopsychiatry, 77(3), 402-418.

Beg, M. R., Casey, J. E., \& Saunders, C. D. (2007). A typology of behavior problems in preschool children. Assessment, 14(2), 111-128. doi:10.1177/1073191106297463

Bracken, B. A., Keith, L. K., \& Walker, K. C. (1998). Assessment of preschool behavior and social-emotional functioning: A review of thirteen third-party instruments. Journal of Psychoeducational Assessment, 16, 153-169.

Breitenstein, S. M., Hill, C., \& Gross, D. (2009). Understanding disruptive behavior problems in preschool children. Journal of Pediatric Nursing, 24(1), 3-12. doi:10.1016/j. pedn.2007.10.007

Briggs-Gowan, M. J., \& Carter, A. S. (2008). Social-emotional screening status in early childhood predicts elementary school outcomes. Pediatrics, 121(5), 957-962. doi:10.1542/ peds. $2007-1948$

Campbell, S. B., Shaw, D. S., \& Gilliom, M. (2000). Early externalizing behavior problems: Toddlers and preschoolers at risk for later maladjustment. Development and Psychopathology, 12, 467-488.

Carney, A. G., \& Merrell, K. W. (2005). Teacher ratings of young children with and without ADHD: Construct validity of two child behavior rating scales. Assessment for Effective Intervention, 30(3), 65-75.

Carter, A. S., Briggs-Gowan, M. J., \& Davis, N. O. (2004). Assessment of young children's social-emotional development and psychopathology: Recent advances and recommendations for practice. Journal of Child Psychology and Psychiatry, 45(1), 109-134. doi:10.1046/j.0021-9630.2003.00316.x

Caselman, T. D., \& Self, P. A. (2008). Assessment instruments for measuring young children's social-emotional behavioral development. Children \& Schools, 30(2), 103-115.

Denham, S. A., \& Burton, R. (2003). Social and emotional prevention and intervention programming for preschoolers. New York: Kluwer Academic.

Epstein, M. H., Synhorst, L. L., Cress, C. J., \& Allen, E. A. (2009). Development and standardization of a test to measure the emotional and behavioral strengths of preschool children. Journal of Emotional and Behavioral Disorders, 17(1), 29-37. doi:10.1177/1063426608319223

Goodman, R. (1997). The Strengths and Difficulties Questionnaire: A research note. Journal of Child Psychology and Psychiatry, 38, 581-586.

Herrera, M., \& Little, E. (2005). Behaviour problems across home and kindergarten in an Australian sample. Australian Journal of Educational \& Developmental Psychology, 5, 77-90.

Kline, P. (2000). Handbook of psychological testing ( $2^{\text {nd }}$ ed.). London: Routledge.

Kline, R. B. (1998). Principles and practice of structural equation modeling. New York: Guilford Press.
Liu, J., Cheng, H., \& Leung, P. W. (2011). The application of the Preschool Child Behavior Checklist and the CaregiverTeacher Report Form to Mainland Chinese children: Syndrome structure, gender differences, country effects, and interinformant agreement. Journal of Abnormal Child Psychology, 39, 251-264. doi:10.1007/s10802-010-9452-8

Lopes, J. A., Machado, M. L., Pinto, A. M., Quintas, M. J., \& Vaz, M. C. (1994). Avaliação de distúrbios de comportamento em crianças de idade pré-escolar. In L. S. Almeida \& I. S. Ribeiro (Eds.), Avaliação psicológica: Formas e contextos (pp. 209-226). Braga, Portugal: Associação dos Psicólogos Portugueses.

Madle, R. A. (2005). Review of the Preschool and Kindergarten Behavior Scales, second edition. In R. Spies \& B. Plake (Eds.), The sixteenth mental measurements yearbook (pp. 813-816). Lincoln, NE: Buros Institute of Mental Measurements, University of Nebraska.

Major, S. O. (2007). Avaliação de aptidões sociais e problemas de comportamento em idade pré-escolar: Estudo exploratório com crianças portuguesas (Dissertação de mestrado, Faculdade de Psicologia e de Ciências da Educação, Universidade de Coimbra, Portugal).

Major, S. O. (2011). Avaliação de aptidões sociais e problemas de comportamento em idade pré-escolar: Retrato das crianças portuguesas (Tese de doutorado, Faculdade de Psicologia e de Ciências da Educação, Universidade de Coimbra, Portugal).

Major, S. O., Seabra-Santos, M. J., \& Merrell, K. W. (2011). Escalas de Comportamento para a Idade Pré-Escolar - $2^{\mathrm{a}}$ edição (ECIP-2). In C. Machado, M. Gonçalves, L. Almeida, \& M. R. Simões (Eds.), Instrumentos e contextos de avaliação psicológica (Vol. 1, pp. 249-268). Coimbra, Portugal: Almedina.

Marinheiro, A. L., \& Lopes, J. A. (1999). Avaliação de problemas de comportamento em idade pré-escolar. Psicologia: Teoria, Investigação e Prática, 4(1), 91-108.

Marzocchi, G. M., Capron, C., Pietro, M. D., Tauleria, E. D., Duyme, M., Frigerio, A., ...Thérond, C. (2004). The use of the Strengths and Difficulties Questionnaire (SDQ) in southern European countries. European Child \& Adolescent Psychiatry, 13(2, Suppl. 2), 40-46. doi:10.1007/s00787-004-2007-1

Merrell, K. W. (1994). Preschool and Kindergarten Behavior Scales. Austin, TX: PRO-ED.

Merrell, K. W. (1995). Relationships among early childhood behavior ratings scales: Convergent and discriminant construct validity of the Preschool and Kindergarten Behavior Scales. Early Education \& Development, 6(3), 253-264.

Merrell, K. W. (1996). Social-emotional problems in early childhood: New directions in conceptualization, assessment, and treatment. Education and Treatment of Children, 19, 458-473.

Merrell, K. W. (2002). Preschool and Kindergarten Behavior Scales - Second edition. Austin, TX: PRO-ED.

Merrell, K. W. (2008). Behavioral, social, and emotional assessment of children and adolescents ( $3^{\text {rd }}$ ed.). New York: Erlbaum.

Merrell, K. W., \& Gimpel, G. A. (1998). Social skills of children and adolescents: Conceptualization, assessment, treatment. Mahwah, NJ: Erlbaum.

Nunnally, J. C. (1978). Psychometric theory (2 ${ }^{\text {nd }}$ ed.). New York: McGraw-Hill.

Tabachnick, B. G., \& Fidell, L. S. (2001). Using multivariate statistics $\left(4^{\text {th }}\right.$ ed.). Boston, MA: Allyn and Bacon.

Recebido: $30 / 04 / 2013$

$1^{a}$ revisão: $23 / 07 / 2013$

Aceite final: $02 / 08 / 2013$ 